\title{
Improving Properties of Polyester and Cellulose Acetate Fabrics using Laser Irradiation
}

\author{
Kamel MM ${ }^{1}$, Raslan WM${ }^{1}$, Helmy $\mathrm{HM}^{1 *}$ and Al-Ashkar $\mathrm{E}^{2}$
}

${ }^{1}$ Textile Research Division, National Research Centre, Cairo, Egypt

${ }^{2}$ Physics Research Division, National Research Centre, Cairo, Egypt

\begin{abstract}
Polyester (PET) and Cellulose Acetate (CA) fabrics were modified by excimer laser irradiation. The induced surface changes were characterized by Scaning Electron Microscopy (SEM). The effect of laser treatment conditions such as power and time of irradiation on the dyeing behaviour of both polyester and cellulose acetate fabrics was studied. A greater depth of shade was achieved on laser treated fabrics compared to the untreated fabric dyed with disperse dye upon the same dyeing conditions. Fabric wettability, surface roughness and fastness properties were also given. Microscopic analysis of the cross section of the dyed samples showed deeper penetration of dye molecules inside the interior structure of cellulose acetate fabric. The depth of disperse dye into CA fibers increased from $20.2 \%$ for untreated sample to $43.9 \%$ for treated one that may help to overcome the ring dyeing problem. Also, laser treatment of cellulose acetate was more effective in enhancing its dyeability with disperse dye than ultrasonic dyeing technique under the same dyeing conditions.
\end{abstract}

Keywords: Polyester; Cellulose acetate; Disperses dye; Laser treatment; Surface modification; Dyeability

\section{Introduction}

Poly Ethylene Terephthalate (PET) was the most important synthetic fibers in terms of annual world demand as well as in terms of end uses. Cellulose Acetate (CA) is considered as man made fibers and it was used in fine clothes. Both PET and CA fabrics were dyed with disperse dyes, which have a very low water solubility and were applied as aqueous dispersions. The dyeing process with disperse dyes can be regarded as four sequential stages [1], (i) dissolution of dye molecules from the dispersed state into the monomolecular dissolved state, (ii) transportation through the bulk solution to near the surface of the fiber, (iii) diffusion through any boundary layer surrounding the fiber and partition to the immediate surface of the fiber, (iv) diffusion within the fiber. The diffusion within the fiber is commonly regarded as the rate-determining step in a circulating dye bath for well formulated dyes. The surface modification of fibers may affect the diffusion step and consequently the colour depth.

Excimer laser etching of polyester fiber surfaces had been reported [2]. The induced physical and chemical properties due to this surface morphological modification had important impacts in textile processing of the fibers. The dyeability was found to be improved significantly after laser treatment. The wetting behaviour of the irradiated fabric also changed substantially. Induced properties of polyester fabric due to irradiation of excimer laser, including surface luster, wettability, dyeability and stability of the resulting micro-structures in the fiber surface, were investigated. After excimer laser treatment, the dyeability of the fabric was improved. The study on surface reflectance showed that excimer laser treatment resulted in a reduction and a more uniform distribution of fabric glossiness; the treated polyester fabric samples had a silk-like appearance [3].

The aim of this work was studying the dyeing behavior of laser treated polyester and cellulose acetate fabrics dyed with disperse dyestuffs. The reflection of the morphological changes induced by excimer laser ablation on the dyeing ability was deduced. The crosssection of dyed fibers was also evaluated. The fiber wettability, surface roughness, whiteness, tensile properties and fastness properties were also indicated. The Chemical Oxygen Demand (COD) after dyeing of CA and PET was evaluated as an indication of reduction of the pollution impacts.

\section{Experimental}

\section{Materials}

White secondary cellulose acetate (CA), satin weave, of density $1.32 \mathrm{~g} / \mathrm{cm}^{3}$ and of $38.5 \%$ acetyl content was used. The fabric was cleaned in an aqueous solution containing $2 \mathrm{~g} / \mathrm{l}$ of nonionic detergent (Hostapal $\mathrm{CV}$, Clariant) at $60^{\circ} \mathrm{C}$ for $20 \mathrm{~min}$ followed by warm and cold rinsing. The fabric was dried under ambient conditions. Polyester fabric (78 dtex, 34 filaments) was provided by Misr Rayon Co, Kafr El-Dawar, Egypt. The fabric was soaped at $40^{\circ} \mathrm{C}$ for $30 \mathrm{~min}$, thoroughly washed, and air dried at room temperature.

Commercial disperse dyes such as C. I. Disperse Red 60 (anthraquinone structure) and C. I. Disperse Red 82 (monoazo structure) were used for dyeing polyester and cellulose acetate respectively. All used chemicals were of reagent grade.

\section{Laser treatment}

Irradiation of polyester and acetate fabric was conducted using a Continuum Laser PRII 8000 OPO (Continuum Electro-Optics, Inc, USA, 2007) pulsed visible excimer laser of wavelength $(\lambda) 532 \mathrm{~nm}$, time of pulse $7 \mathrm{~ns}$ and maximum power of $5000 \mathrm{~m}$ watt Continuous Waves (CW) laser treatment was carried out using Infra Red Diode Laser of wave length $(\lambda) 807 \mathrm{~nm}$ and maximum power of $457 \mathrm{~m}$ watt and

*Corresponding author: Helmy HM, Textile Research Division, National Research Centre, Cairo, Egypt, E-mail: hany_helmy2001@yahoo.com

Received April 16, 2012; Accepted June 23, 2012; Published June 25, 2012

Citation: Kamel MM, Raslan WM, Helmy HM, Al-Ashkar E (2012) Improving Properties of Polyester and Cellulose Acetate Fabrics using Laser Irradiation. J Textile Sci Eng 2:117. doi:10.4172/2165-8064.1000117

Copyright: (c) 2012 Kamel MM, et al. This is an open-access article distributed under the terms of the Creative Commons Attribution License, which permits unrestricted use, distribution, and reproduction in any medium, provided the original author and source are credited. 
intensity of $50 \mathrm{watt} / \mathrm{cm}^{2}$. The applied powers varied from $110-510 \mathrm{mw}$ for different time intervals varied from 1 to $10 \mathrm{~min}$. The fabric samples were white plain weaves. All the samples were washed before excimer laser treatment to eliminate oil and other contamination on the surface.

\section{Dyeing}

Polyester and cellulose acetate fabrics were exhaust dyed using C. I. Disperse Red 60 and C.I Disperse Red 82 respectively. Dyeing of the fabrics was carried out in a bath containing (1\% o.w.f) dye solution at different temperatures $\left(70^{\circ}-100^{\circ} \mathrm{C}\right)$ for different time intervals $(5-90$ min) using a liquor ratio of 1:50. Dispersing agent of $1 \mathrm{~g} / \mathrm{l}$ was added. The $\mathrm{pH}$ of the bath was adjusted at $4.5 \mathrm{using}$ acetic acid. At the end of the dyeing, the dyed samples were rinsed with hot and cold water and air dried.

Ultrasonic dyeing: Ultrasonic dyeing was carried out using Digital Ultrasonic Cleaner SH 400 (MTI Corporation, USA). The experimental device was composed of an electrical generator operated at a frequency of $50 \mathrm{kHz}$ and a power of $400 \mathrm{w}$. The samples, weighing $1.0 \mathrm{~g}$, were dyed in $50 \mathrm{ml}$ beakers with C.I Disperse Red 82. The amount of dye used was $1 \%$ o.w.f. at a liquor ratio of $1: 50$. The dyeing was carried out at $70^{\circ} \mathrm{C}$ and $\mathrm{pH}$ of dye liquors were adjusted to 4.5 .

\section{Measurements}

Whiteness: Changes in fabric whiteness after laser treatment were measured using Ultra Scan PRO-Hunter Lab spectrophotometer according to AATCC test method 153-1985 [4].

Wettability: The Wettability was evaluated by measuring the wetting time according to the AATCC test method 35-1989 [5]. A drop of water was allowed to fall from a fixed height onto the surface of the sample under examination. The taken time for disappearing of the drop of water was measured and expressed by the wetting time. The average value of five readings was taken as a result.

Surface roughness: Changes in roughness values were measured for treated and untreated samples using a surface roughness measuring instrument SE 1700 a (Japan). The results were the average values of five readings.

Tensile strength: Fabric tensile strength test was conducted according to ASTM method 1682 (1994), which is considered as a standard method for breaking force and elongation of tensile fabrics [6]. The width and the length of the fabric strip were $50 \mathrm{~mm}$ and 200 $\mathrm{mm}$ respectively. The average of five samples readings was considered.

Scanning electron microscopy (SEM): The surface morphology of untreated and laser treated fabric was investigated by using SEM, JSMT-20, JEOL-Japan.

Color measurements: The color intensity, expressed as K/S value of the dyed samples was determined spectrophotometrically using Ultra Scan PRO-Hunter Lab spectrophotometer and was estimated by applying the Kubelka Munk equation [7]. The K/S value of each sample was measured five times and the average result was recorded.

Fastness properties: The dyed samples were washed using $2 \mathrm{~g} / \mathrm{l}$ nonionic detergent (Egyptol PLM) at $40^{\circ} \mathrm{C}$ for $30 \mathrm{~min}$, and tested against fastness properties using standard ISO test methods. The color fastness to washing was determined according to the test method ISO 105-C04 (1989) [8]. The light fastness test was evaluated according to the test method ISO 105-B02 (1988) [9].
Preparation of cross-sections: The dyed fibers were mounted in blocks and trimmed to obtain one clean cut surface perpendicular to the fiber axis. The untreated and treated CA and PET samples were subjected to image microscopic analysis. The distance that the dye had diffused into the fiber was determined, starting from the fiber surface along the fiber radius. The average of 15 readings was considered. The penetration depth $\%$ was evaluated for each sample.

\section{Results and Discussion}

\section{Whiteness, wettability and surface roughness}

The whiteness index: The whiteness index of both untreated and treated fabric was measured. It can be noticed from Table 1 that there was a slight increase in whiteness of both CA and PET fabrics after laser treatment. The whiteness indexes of pulsed laser treated CA samples at 310 and $320 \mathrm{mw}$ were approximately similar and it slightly decreased by increasing the laser power up to $413 \mathrm{mw}$. Pulsed laser treatment of PET samples at different powers $(110-510 \mathrm{mw})$ had no significant effect on the whiteness of the fiber while CW laser treatment gives a slight increase in W.I. as shown in Table 1.

The wettability of both untreated and laser treated PET and CA samples were evaluated by measuring the wetting time (sec). Table 1 illustrates the values of the wetting times for different CA and PET samples treated at different powers. It is clear that the drastic increase in wettability was achieved within one min upon applying laser irradiation. All treated samples have higher wettability than the untreated one. The lower power level of laser irradiation was more effective in enhancing the fiber wettability than the higher power. This holds true as reported elsewhere [10] that the high energy dose treatment of PET resulted in an increased contact angle indicating that the surface was more hydrophobic while the low energy treatment decreases the contact angle resulting in more hydrophilic fiber surface. It was also reported [10] that the $\mathrm{O} / \mathrm{C}$ ratio increased significantly after laser treatment of PET. So, the fiber surface has higher oxygen content than before irradiation resulting in increasing the hydrophilicity of the fiber due to a subsequent reaction of laser irradiated surface with the atmospheric oxygen during the treatment. It seemed likely that the water penetration into the fibrous assembly is dependent on the wettability of the fiber surface as a capillary wall $[11,12]$. So, the moisture absorbing property and consequently the dyeability for treated fabric will be enhanced as will be shown later (Figures 3-6).

\begin{tabular}{|l|l|l|l|}
\hline Type of samples & $\begin{array}{l}\text { Whiteness } \\
\text { index (W. I. })\end{array}$ & Wettability $(\mathrm{sec})$ & $\begin{array}{l}\text { Surface roughness } \\
(\mu \mathrm{m})\end{array}$ \\
\hline -Untreated CA & 88.2 & 3.0 & 23.75 \\
\hline $\begin{array}{l}\text {-Pulsed laser treated } \\
\text { CA for 1 min at: } 310 \\
\text { mw }\end{array}$ & 90.8 & 1.71 & 26.46 \\
\hline $320 \mathrm{mw}$ & 90.78 & 1.71 & 26.47 \\
\hline $413 \mathrm{mw}$ & 90.0 & 1.85 & 26.5 \\
\hline -Untreated PET & 121.9 & 7.6 & 18.31 \\
\hline $\begin{array}{l}\text {-Pulsed laser treated } \\
\text { PET for 1 min at: } 110 \\
\text { mw }\end{array}$ & 122.0 & 5.3 & 20.29 \\
\hline \begin{tabular}{l}
$195 \mathrm{mw}$ \\
\hline $510 \mathrm{mw}$
\end{tabular} & 122.2 & 5.5 & 20.36 \\
\hline $\begin{array}{l}\text { CW *laser treated PET } \\
\text { for 1 min at 160 mw }\end{array}$ & 122.3 & 5.5 & 20.61 \\
\hline
\end{tabular}

${ }^{*} \mathrm{CW}$ (Continues Wave), $\lambda=807 \mathrm{~nm}$

Table 1: Whiteness index, wettability and surface roughness of laser treated CA and PET fabrics. 
Citation: Kamel MM, Raslan WM, Helmy HM, Al-Ashkar E (2012) Improving Properties of Polyester and Cellulose Acetate Fabrics using Laser Irradiation. J Textile Sci Eng 2:117. doi:10.4172/2165-8064.1000117

The surface roughness: The surface roughness of untreated and treated CA and PET samples with laser irradiation at different powers for $1 \mathrm{~min}$ is measured. The results are given in Table 1. The surface roughness for the treated CA and PET samples were higher than the untreated one. Also, it increased gradually with increasing the irradiation power. It can be also seen that pulsed visible excimer laser is slightly more effective on roughness property than continuous laser treatment. The surface roughness may be due to ablation effect of laser treatment.

Tensile strength and elongation \% at break: The tensile strength and elongation \% at break of untreated CA sample were $35.6 \mathrm{~kg}$ and $24 \%$ respectively compared to $34.3 \mathrm{~kg}$ and $20 \%$ for laser treated sample at $310 \mathrm{mw}$ for $1 \mathrm{~min}$. These values were $56.8 \mathrm{~kg}$ and $48 \%$ for untreated polyester fabric compared to $53 \mathrm{~kg}$ and $45 \%$ for laser treated sample at $160 \mathrm{mw}$ for $1 \mathrm{~min}$. It could be noticed that there was a slight decrease in tensile properties of both laser treated CA and PET samples. Therefore, it seems that the laser impact damage to the fabric is negligibly small (Table 2).

Surface Morphology: The surface morphology of untreated and laser treated cellulose acetate and polyester fabrics was characterized by SEM. Figures 1(a) and 2(a) show the micrographs of untreated $\mathrm{CA}$ and PET respectively, while Figures $1(\mathrm{~b}, \mathrm{c})$ and $2(\mathrm{~b}, \mathrm{c})$ represent the micrographs of treated CA and PET respectively at different laser treatment conditions. It can be shown from Figures $1 \mathrm{a}$ and $1 \mathrm{~b}$ that the surface morphology of CA changed after laser treatment. Small ripples appeared on the fiber surface treated with laser at both 310 and 320 mw for 1 min compared with smooth surface for untreated one Figures 1(a). These ripples-like structure affect some properties of the fiber such as wettability and dyeing ability; darker shades were obtainable on laser treated fabrics [11] as will be shown later.

On the other hand, polyester fabrics were treated with continuous waves $(\mathrm{CW})$ laser irradiation of $160 \mathrm{mw}$ at different times (1and 10 $\mathrm{min}$ ) and the surface morphology of these samples was characterized by SEM. It can be shown from Figures $2(b, c)$ that there were some cracks and ripples appeared on the fiber surface and these cracks became deeper with prolonged exposure to laser irradiation. Laser treatment created a degree of roughness on the fiber surface, resulting in an enhancement of the surface area and diffusion reflection and consequently improving the dye ability.
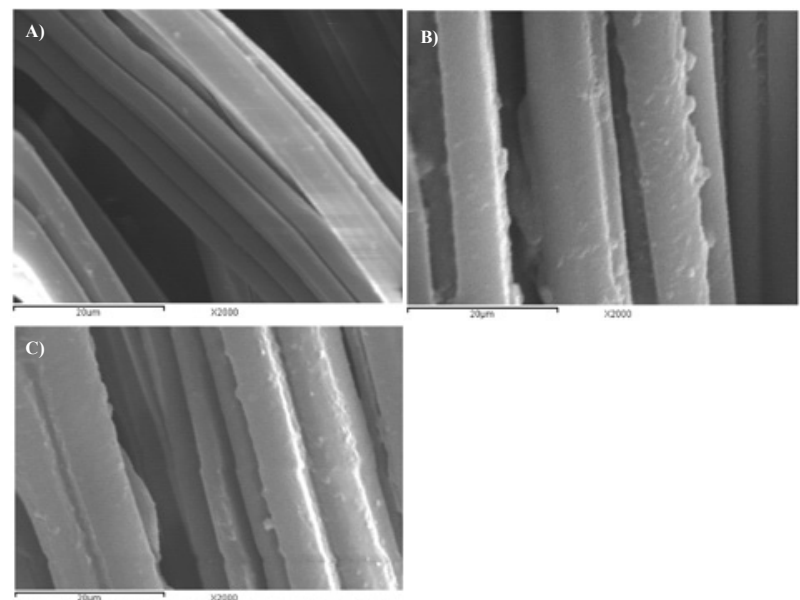

Figure 1: A) SEM of untreated CA. B) SEM of pulsed laser treated CA fibres at $310 \mathrm{mw}$ for $1 \mathrm{~min}$. C) SEM of pulsed laser treated CA fibres at $320 \mathrm{mw}$ for $1 \mathrm{~min}$
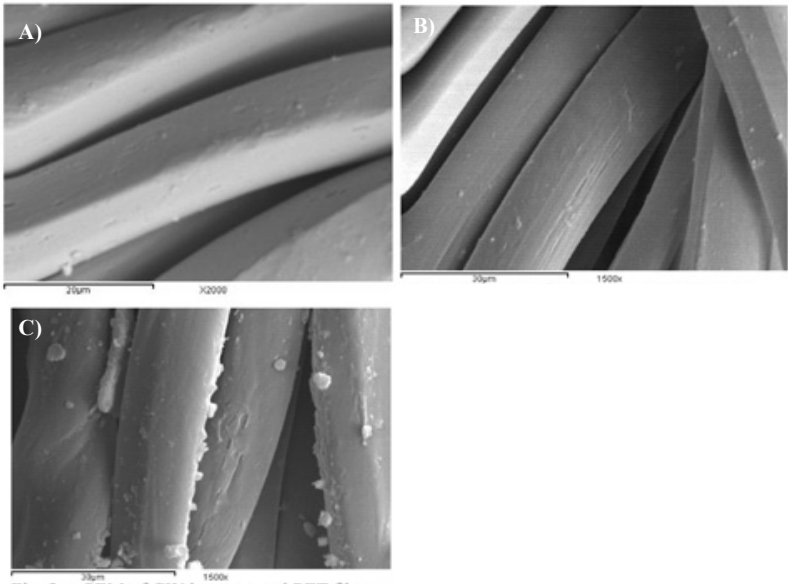

Figure 2: A) SEM of untreated PET fibres. B) SEM of CW laser treated PET fibres at $160 \mathrm{mw}$ for $1 \mathrm{~min}$. C) SEM of CW laser treated PET fibres at $160 \mathrm{mw}$ for $10 \mathrm{~min}$.

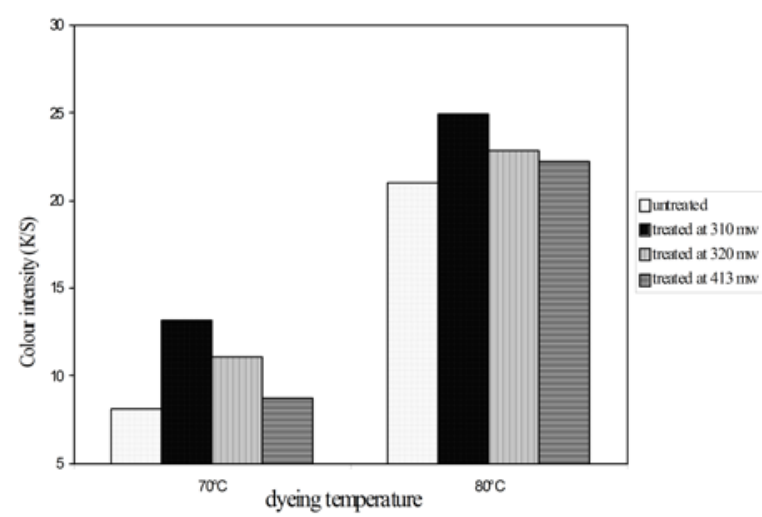

Figure 3: Dependence of the color intensity of pulsed laser treated CA fabric on the laser power at different dyeing temperatures.

Treatment: $1 \mathrm{~min}$

Dyeing conditions: 1\% (o.w.f) C.I. Disperse Red 82, 30 min, pH 4.5, liq. ratio 1:50

Dyeing behavior: Both CA and PET were disperse dyes. There were no polar molecules and pendent groups in polyester fibers, and therefore the dyeability of polyester was poor compared to those of other synthetic fibers. The excimer UV-laser treatment can improve the dye ability of polyester fibers [3]. Figures 3-5 represented the color intensity of laser treated CA fabric. The results showed that the pulsed excimer laser treatment brought out faster dye processing and higher colour intensity. Figure 3 showed the dependence of the colour intensity on the laser power at different dyeing temperatures $\left(70^{\circ}\right.$ and $\left.80^{\circ} \mathrm{C}\right)$. As the laser power increased the color intensity decreased which may be due to the rapid heating caused by the absorption of energy from the laser beam which led to breaking of some of the fibers, forming some fragments which remained adhered to the fiber surface. As a result, the amount of dyes absorbed by laser treated samples was decreased and resulted in lower color strength when laser power increased. But the color intensity for the treated samples is still higher than the untreated one. Figures 4 showed the color intensity of pulsed laser treated CA fabric at $310 \mathrm{mw}$ for $1 \mathrm{~min}$ and dyed at $80^{\circ} \mathrm{C}$ in relation to the dyeing time. The colour intensity for the laser treated CA was higher than the untreated one. It could be seen that the maximum colour intensity of 
Citation: Kamel MM, Raslan WM, Helmy HM, Al-Ashkar E (2012) Improving Properties of Polyester and Cellulose Acetate Fabrics using Laser Irradiation. J Textile Sci Eng 2:117. doi:10.4172/2165-8064.1000117

Page 4 of 6

untreated one could be attained at shorter time upon applying the laser treatment. The half dyeing time $\left(t_{1 / 2}\right)$ which was the time required for the fabric to take up half of the amount of dye (at equilibrium) was estimated from the isotherm directly (Figures 4 ). It is clear that $t_{1 / 2}$ decreased from about $10 \mathrm{~min}$ for untreated sample to $7 \mathrm{~min}$ for laser treated one. The main factor which explained the improvement of the dyeability of the irradiated CA fabric was the increase of the overall surface area as a result of the morphological modification induced by excimer laser (Figures 1a-c).

Ultrasonic energy was used in textiles to speed and develop the textile processes. Figures 5 shows the dyeability of laser treated CA fabric compared to ultrasonic dyeing technique at $70^{\circ} \mathrm{C}$. Results have shown that ultrasonic energy has affected the colour intensity positively. Dye transfer to the fabric after ultrasonic dyeing was more than the conventional one (untreated sample). The intensity of dyeing follows the order; laser treated fabric, ultrasonic dyed sample, untreated one.

Treatment of Polyester fabric with pulsed laser irradiation at 110 , 195 and $520 \mathrm{mw}$ had no remarked effect on its dyeability. So, polyester samples were treated with CW laser powers at $160 \mathrm{mw}$ and then dyed with C.I. Disperse dye 60 at $100^{\circ} \mathrm{C}$. The effect of laser treatment on the dyeability of polyester fabric is shown in Figure 6. The results show that the dyeing behaviour of laser treated PET was enhanced. The K/S values of laser irradiated samples were higher than the corresponding untreated samples. Figure 6 shows the dyeing ability of CW laser treated PET fabric at $160 \mathrm{mw}$ for different exposure time (1-10 min). All samples treated under these conditions show higher K/S values than the untreated one. It was clear that K/S values were inversely proportional with exposure time. It was also, noticed that $t_{1 / 2}$ of treated samples at $160 \mathrm{mw}$ for $1 \mathrm{~min}$ was about $11 \mathrm{~min}$ compared to $18.5 \mathrm{~min}$ for untreated one. It was assumed that the greater depth of shade was caused by the scattering of light owing to the modification on the fiber surface observed by SEM. The deeper shade might be also due to greater dye uptake by the fiber as a result of increasing the surface area.

Monitoring dye penetration: Figures $7 \mathrm{a}$ and $\mathrm{b}$ showed the cross-

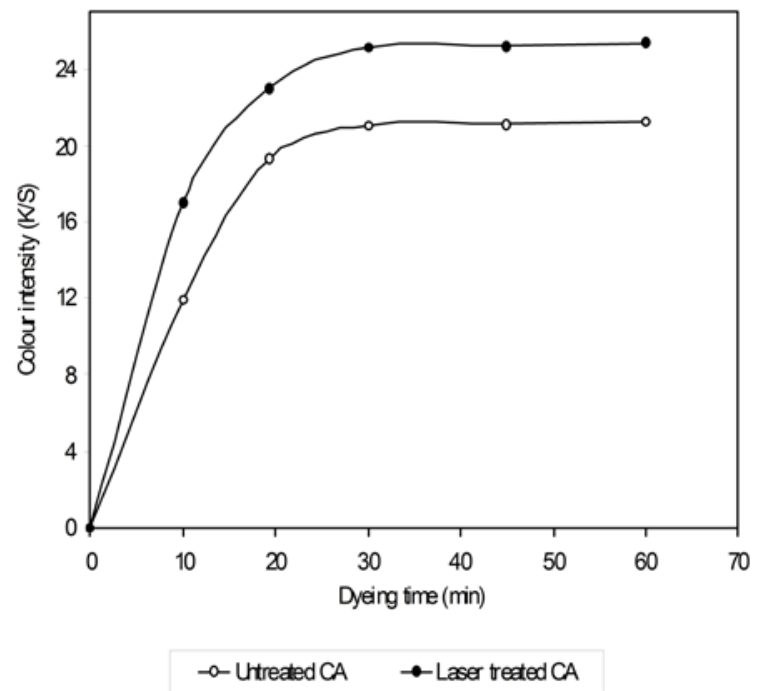

Figure 4: The color intensity of laser treated CA fabric in relation to the time of dyeing.

Treatment: $310 \mathrm{mw}, 1 \mathrm{~min}$

Dyeing conditions: $1 \%$ (o.w.f) C.I. disperse Red 82, 80 C, pH 4.5, Liq. Ratio 1:50

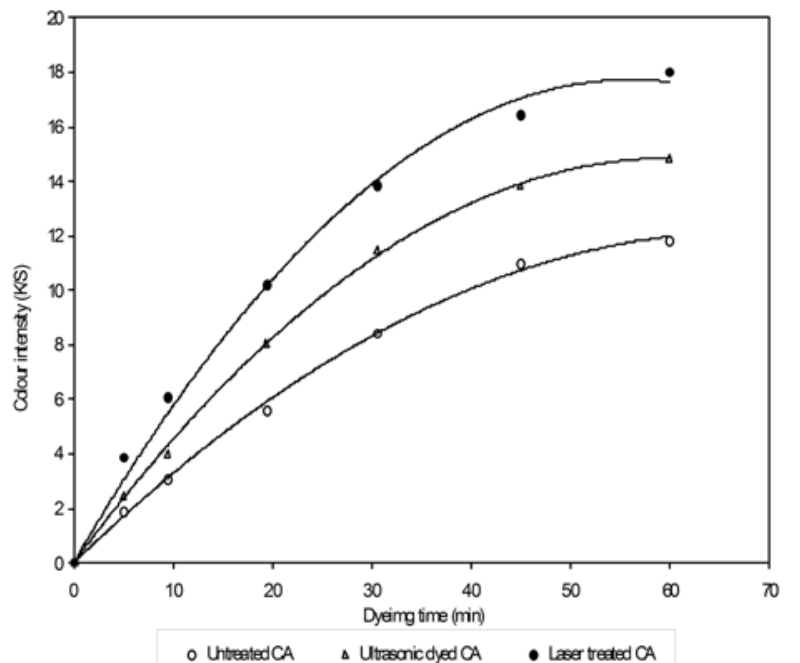

Figure 5: Dyeability of laser treated CA fabric compared to ultrasonic dyed samples.

Treatment: $310 \mathrm{mw}, 1 \mathrm{~min}$

Dyeing conditions: $1 \%$ (o.w.f) C.I. Disperse $\operatorname{Red} 82,70^{\circ} \mathrm{C}, \mathrm{pH} 4.5$, liq. ratio $1: 50$

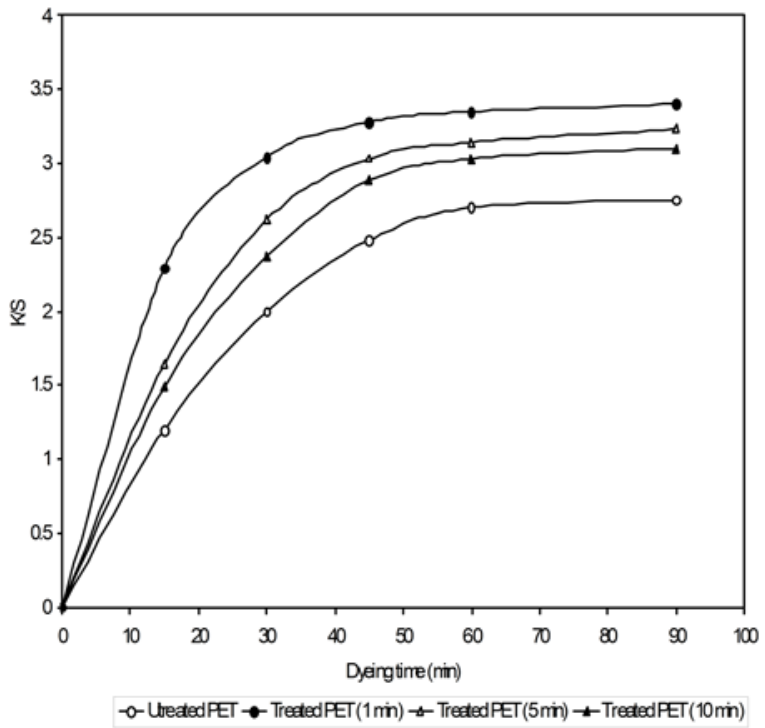

Figure 6: Dyeability of CW laser treated PET fabric at different exposure time. Treatment : CW, $160 \mathrm{mw}, \lambda=807 \mathrm{~nm}$.

Dyeing conditions : $1 \%$ (o.w.f) Disperse Red $60,100^{\circ} \mathrm{C}$, pH 4.5, liq. Ratio 1:50.

sections of untreated and pulsed laser treated CA fibers at $310 \mathrm{mw}$ for $1 \mathrm{~min}$ and dyed with $1 \%$ o.w.f. C. I. Disperse Red 82 at $80^{\circ} \mathrm{C}$ for 60 min respectively. This allowed studying dye penetration profiles within single fibers in detail as needed for a better understanding of the dyeing process. Both figures clearly showed the penetration of dye in the fiber. The penetration depth was the most suitable quantifiable parameter for the dye diffusion behavior in the fibers. It can be noticed that the depth of the dye in the interior structure of the fiber increased as a result of laser treatment. The mean depth \% of the disperse dye inside the laser treated CA fiber was found to be more than that of the untreated dyed one. It was evaluated by $43.9 \%$ compared to $20.2 \%$ for untreated one. While Figures $8 \mathrm{a}$ and $\mathrm{b}$ show the cross-sections of untreated and CW laser treated PET fibers at $160 \mathrm{mw}$ for $1 \mathrm{~min}$ and dyed with $1 \%$ o.w.f. C. 
Citation: Kamel MM, Raslan WM, Helmy HM, Al-Ashkar E (2012) Improving Properties of Polyester and Cellulose Acetate Fabrics using Laser Irradiation. J Textile Sci Eng 2:117. doi:10.4172/2165-8064.1000117

Page 5 of 6

I. Disperse Red 60 at $100^{\circ} \mathrm{C}$ for 60 min respectively. The mean depth $\%$ is evaluated by $\sim 30 \%$ for treated sample compared to $18.5 \%$ for untreated one. However, it is important to realize that the penetration during the dyeing cycle gave vital information on the diffusion behaviour during a common industrial dyeing cycle that cannot be easily achieved by diffusion coefficient measurements. It was also reported elsewhere that a mean dye depth $<30 \%$ inside the fiber is defined as ring dyeing [1319].

Fastness properties: The light fastness of both untreated and treated samples for the two psubstrates (PET and CA) was evaluated. It was found that the light fastness values for untreated PET and CA were 5 and 6 respectively compared to 6 and 7 for treated samples (Table 3 ). While the washing fastness for both substrates were found to be unchanged by laser treatment.

Chemical oxygen demand (COD): Waste water treatment requirements for textile and other industries have outstripped the current methods available for reducing COD. Figures 9 showed COD of the bath after dyeing CA and PET fabrics with disperse dyes. It was clear that laser treatment for both CA and PET fabrics reduced the COD of the effluents from the dyeing process and at the mean time enhanced the dyeability and fastness properties. It can be also noticed
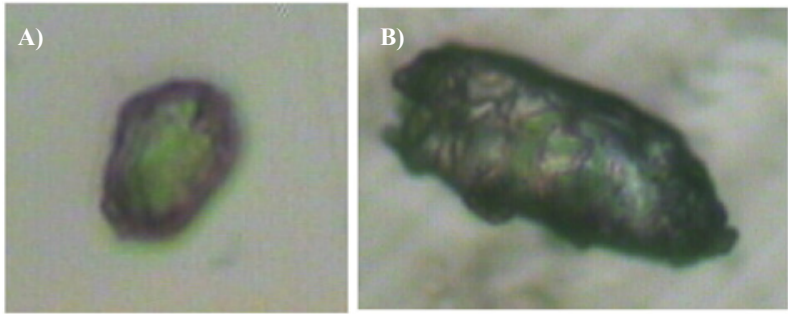

Figure 7: A) Cross section of untreated CA. B) Cross section of pulsed laser treated CA at $310 \mathrm{mw}$ for $1 \mathrm{~min}$.

Dyeing condition: $1 \%$ (o.w.f) C.I. Disperse Red $82,80^{\circ} \mathrm{C}, 60 \mathrm{~min}, \mathrm{pH} 4.5$, liq. Ratio 1:50
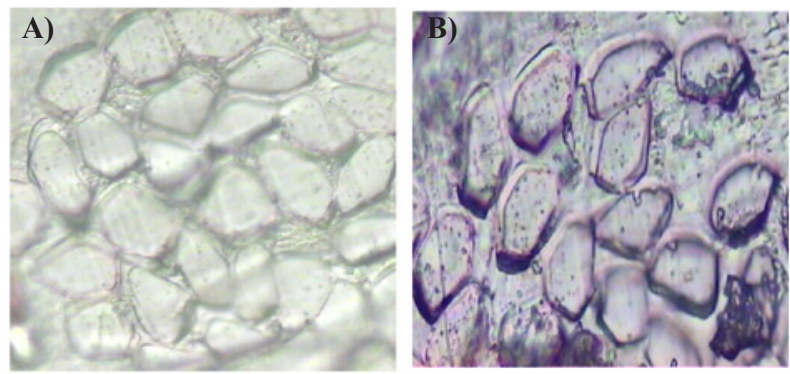

Figure 8: A) Cross section of untreated Pet fabrics. B) Cross section of CW laser treated PET fabrics at $160 \mathrm{mw}$ for $1 \mathrm{~min}$.

Dyeing conditions: $1 \%$ (o.w.f) C.I. Disperse Red $60,100^{\circ} \mathrm{C}, 60 \mathrm{~min}, \mathrm{pH} 4.5$, liq Ratio 1:50

\begin{tabular}{|l|l|l|}
\hline Samples & Tensile strength $(\mathrm{kg})$ & Elongation \% \\
\hline -Untreated CA & 35.6 & 24 \\
-Pulsed laser treated CA at $310 \mathrm{mw}$ & 34.3 & 20 \\
\hline -Untreated PET & 56.8 & 48 \\
-CW laser treated PET at $160 \mathrm{mw}$ & 53 & 45 \\
\hline
\end{tabular}

Laser treatment: $1 \mathrm{~min}$

Table 2: Tensile strength and elongation at break of laser treated CA and PET fabrics.

\begin{tabular}{|l|l|l|}
\hline Samples & Light fastness & $\begin{array}{l}\text { Washing fastness } \\
\mathrm{S}^{*} \mathrm{~S}^{* *} \mathrm{~S}^{* * *} \text { Alt }\end{array}$ \\
\hline Untreated CA & 6 & $4-5433$ \\
\hline Treated CA & 7 & $4-5433$ \\
\hline Untreated PET & 5 & $4-544-54$ \\
\hline Treated PET & 6 & $4-544-54$ \\
\hline
\end{tabular}

\section{*Stain on cotton}

** Stain on wool

*** Stain on sample

Alt (alteration)

Table 3: Fastness properties of laser treated CA and PET.

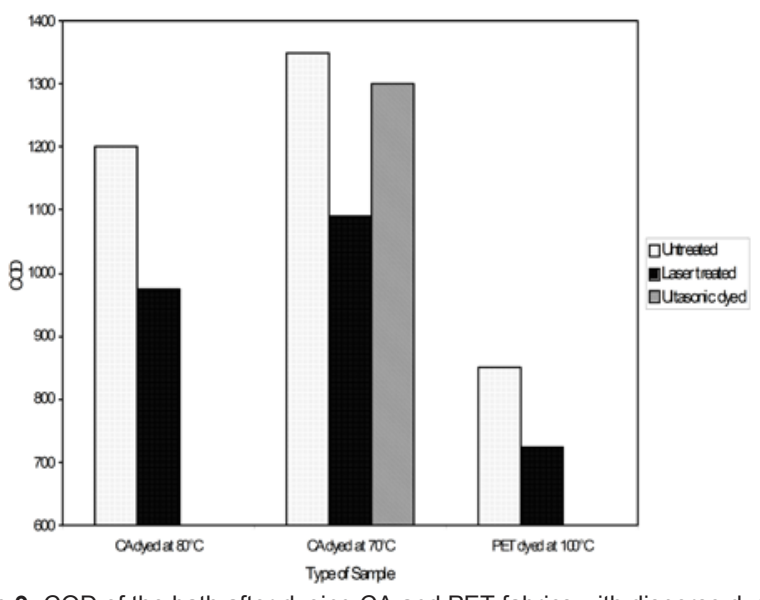

Figure 9: $C O D$ of the bath after dyeing $C A$ and PET fabrics with disperse dyes. Treatment : CA: pulsed laser $310 \mathrm{mw}, 1 \mathrm{~min}$; PET:CW laser at $160 \mathrm{mw}, 1 \mathrm{~min}$

that laser treatment of CA is more effective in reducing COD than ultrasonic dyeing technique. This may be a way to get cleaner product and reduced the pollution impacts.

\section{Conclusion}

This study was concerned to improve the dyeability of CA and PET fabrics with disperse dyes by the pretreatment with laser irradiation. The laser treatment was found to increase the wettability, whiteness index and roughness of the fiber surfaces. This resulted in the enhancement of the fiber dyeability as well as the fastness properties. Laser treatment of both fabrics may overcome the ring dyeing phenomenon as it increased the depth of dye inside the fiber structure over $30 \%$. From the tensile properties results, the laser impact damage to semi-synthetic (CA) and synthetic (PET) textile fabrics was confirmed to be negligibly small.

\section{References}

1. Brennan CM, Bullock JF (1996) Advances in color chemistry, In: Peters AT, Freeman HS (Eds). Physico-Chemical Principles of Color Chemistry. vol. 1 Glasgow: Chapman and Hall.

2. Bahners T, Schollmeyer E (1989) Morphological changes of the surface structure of polymers due to excimer laser radiation: A synergetic effect? J Appl Phys 66: 1884-1886.

3. Lau KS, Chan Pw, Yeung KW, Chan K, Gong WZ (1997) Surface properties of polyester fabrics induced by excimer laser processing. J Mater Process Tech 63: 524-528.

4. AATCC (1985) Technical Manual, Test Method 153.

5. AATCC (1989) Technical Manual, Test Method 35

6. ASTM (1994) Standard Test Method 1682. 
Citation: Kamel MM, Raslan WM, Helmy HM, Al-Ashkar E (2012) Improving Properties of Polyester and Cellulose Acetate Fabrics using Laser Irradiation. J Textile Sci Eng 2:117. doi:10.4172/2165-8064.1000117

7. Judd D, Wyszecki G (1975) Color in Business, Science and Industry. John Wiley, New York

8. ISO 105-C04: (1989) Textiles-Tests for colour fastness-Part C04: Colour fastness to washing: Test 4 .

9. ISO 105-B02: (1988) Textiles-Tests for colour fastness-Part B02: Colour fastness to artificial light: Xenon arc fading lamp test.

10. Wong W, Chan K, Yeung KW (2001) Chemical modification of poly (ethylene Terephthalate) induced by laser treatment. Text Res J 71: 117-120.

11. Yip J, Chan K, Sin KM, Lau KS (2002) Study of physico-chemical surface treatments on dyeing properties of polyamides. Part 1: Effect of tetrafluoromethane low temperature plasma. Color Technol 118: 26-30.

12. Gotoh K, Hayashiya M (2008) Improvement of serviceability properties of synthetic textile fabrics using $172 \mathrm{~nm}$ ultraviolet excimer lamp. Text Res $\mathrm{J} 78$ : 37-44

13. Aspland JR (1997) Textile dyeing and coloration., AATCC, USA.
14. Nunn DM (1979) The dyeing of synthetic-polymer and acetate fibres, Dyers Co Publication Trust: England.

15. Knittel D, Schollmeyer E (1998) Surface structuring of synthetic polymers by UV-laser irradiation. Part IV. Applications of excimer laser induced surface modification of textile materials. Poly Int 45: 110-117.

16. Kan CW (2008) Effects of laser irradiation on polyester textile properties. J App Poly Sci 107: 1584-1589.

17. Watanabe H, Takata T (1996) Surface modification of synthetic fibers by excimer laser irradiation. Die Angewandte Makromolekulare Chemie 235: 95110.

18. Knittel D, Schollmeyer E (1998) Surface structuring of synthetic fibres by UV laser irradiation. Part III. Surface functionality changes resulting from excimerlaser irradiation. Poly Int 45: 103-109.

19. Knittel D, Kesting W, Schollmeyer E (1997) Surface structuring of synthetic fibres by UV laser irradiation, Part I: phenomenological report. Poly Int 43: 231239 